\title{
Gait Enhancing Mobile Shoe (GEMS) for Rehabilitation
}

\author{
Allison de Groot* \\ Biomedical Engineering Department \\ Virginia Commonwealth University
}

\author{
Ryan Decker ${ }^{\dagger}$ \\ Kyle B. Reed \\ Laboratory for Computational Sensing and Robotics \\ Johns Hopkins University
}

\begin{abstract}
Individuals with certain types of central nervous system damage, such as stroke, have an asymmetric walking gait. Using a splitbelt treadmill, where each leg walks at a different speed, has been shown to help rehabilitate walking impaired individuals, but there is one distinct drawback; the corrected gait does not transfer well to walking over ground. To increase the gait transference to another environment, we designed and built a passive shoe that admits a motion similar to that felt when walking on a split-belt treadmill. Our gait enhancing mobile shoe (GEMS) alters the wearer's gait by causing one foot to move backward during the stance phase while walking over ground. No external power is required since the shoe mechanically converts the wearers downward and horizontal forces into a backward motion. This shoe would allow a patient to walk over ground while experiencing the same gait altering effects as felt on a split-belt treadmill, which should aid in transferring the corrected gait to walking in natural environments.
\end{abstract}

Keywords: Medical, Gait rehabilitation, Human performance

\section{INTRODUCTION}

Stroke victims and other individuals who have gait impairments often lack a complete stride while walking. A correct gait involves each leg moving in a similar, but $180^{\circ}$ out of phase, trajectory from the front to the back of the body. Specifically, in stroke patients, one leg does not travel far enough behind the body, which is necessary for efficient gait.

One method for gait correction in an impaired individual is to use a split-belt treadmill $[19,7,24,20]$. Such a system enables independent control of the treads each leg walks on, which allows one leg to effectively move faster than the other. Through repeatedly walking with different speed treads, a split-belt treadmill can alter the gait cycle and temporarily restore normal gait to walking impaired individuals $[14,9,15]$. This effect occurs on a powered treadmill, but unfortunately does not transfer to walking over ground. A person who has adapted to different speed treads will show nearly no improved gait when they transition to overground walking.

Walking involves a context awareness [5] to account for likely perturbations, much like preplanning trajectories when reaching for objects $[1,4,6]$. One example of a similarly incorrect context shift occurs when you step onto an escalator that is not moving. Over the many previous repetitions of getting on and off escalators, your body has developed a model of the dynamics that will occur when your foot first touches the escalator. When your body is not pulled forward as your internal model expects, you will likely stumble slightly, even though you were fully aware that the escalator was not moving before getting on. This is the same context awareness that prevents the learned walking patterns on the treadmill from

*e-mail: degrootay@vcu.edu

†e-mail: ryandecker@jhu.edu

†e-mail: reedkb@jhu.edu transferring to walking over ground, a different context. It is this context awareness that our shoe seeks to overcome.

Our Gait Enhancing Mobile Shoe (GEMS), shown in Figure 1, improves upon the split-belt treadmill method of rehabilitation by allowing one foot to move relative to the ground while overground walking. The shoe is passive and converts the wearer's own downward and horizontal forces into a backward motion. The resulting motion is similar to that of a split-belt treadmill, but with several advantages. The shoe requires no power to operate because all forces are derived from the wearer. The shoe is portable, so it can be used in more locations, including the comfort and convenience of one's home. Using such a device in more locations will likely help the wearer to transfer a correct gait pattern to walking over ground, which is the typical location the shoe would be used. The portability will also allow extended use of the gait altering device, which is likely to produce better rehabilitation effects.

\section{BACKGROUND}

\subsection{Phases and Forces During Walking}

Four phases of walking relevant to the GEMS design are shown in Figure 2, starting with initial heel contact. After the wearer's heel touches the ground, the wearer will move forward as their entire foot comes into contact with the ground. With their foot flat on the ground, their body will pivot around their foot, much like an inverted pendulum [23]. Their body will continue moving forward as their heel rises and they propel themselves forward with the front of their foot. As they are pushing and preparing to lift their foot off the ground, their other leg will initiate this process starting with heel contact.

Our design uses the changing forces throughout the step $[11,2]$ to cause the desired motion. Figure 3 shows the horizontal $\left(F_{x}\right)$ and vertical $\left(F_{z}\right)$ ground reaction forces from one foot while walking. At initial heel contact, the forward force is near $-100 \mathrm{~N}$, which indicates that the leg is actually pushing against the walking direction. Throughout the stance phase, the horizontal force will transition to a similar, but positive, force pushing the person forward. The vertical force is fairly constant near $600 \mathrm{~N}$ during the stance phase since the foot is supporting the wearer's weight.

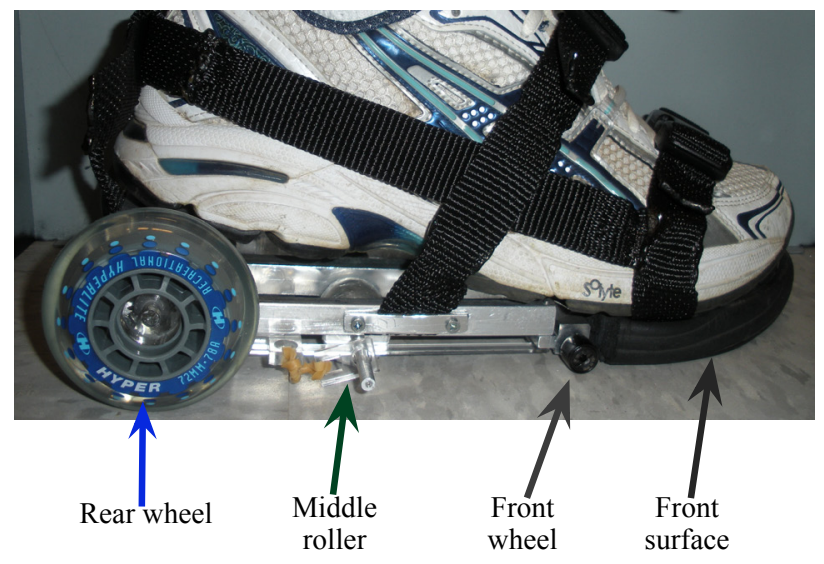

Figure 1: Prototype of the Gait Enhancing Mobile Shoe (GEMS). 


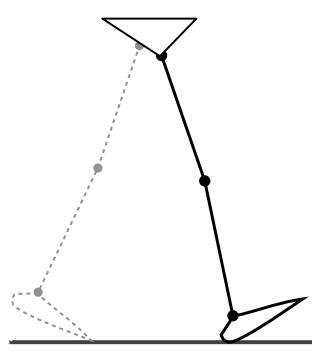

(a) Initial heel contact

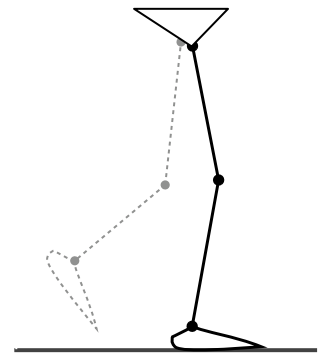

(b) Mid-stance

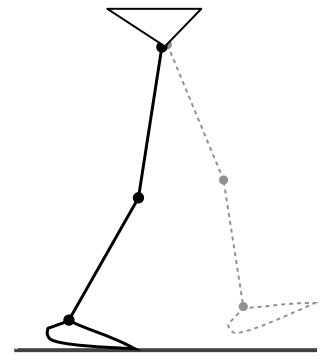

(c) Heel rise

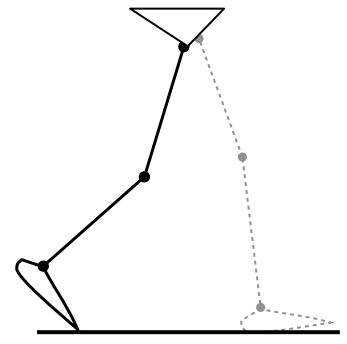

(d) Toe off

Figure 2: The solid line demonstrates one leg during the four phases of motion relevant to this design. Figures redrawn based on [21].

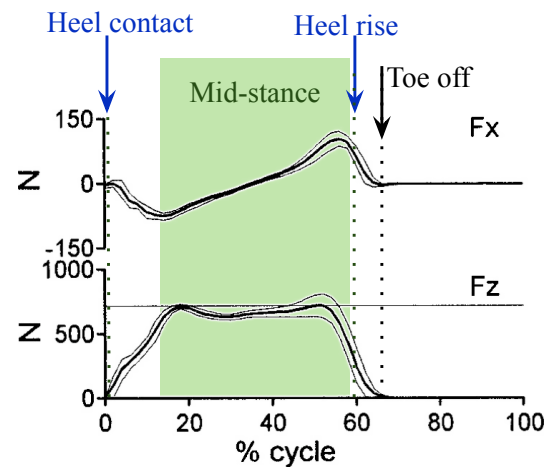

Figure 3: The horizontal $\left(F_{x}\right)$ and vertical $\left(F_{z}\right)$ forces change throughout the gait cycle. The GEM shoe uses these changing forces to passively alter gait patterns for rehabilitation. The figure is modified and used with permission from [2].

Walking inherently relies upon ground friction for stability. For example, walking on ice limits the amount of horizontal force one can apply and can lead to falls. Redfern et al. [12] provide a summary of slipping biomechanics and recovery methods. For our shoe design, it is important to limit the total amount of motion relative to the ground so the recovery reflexes are not activated.

\subsection{Correcting Gait}

Stroke patients often fail to bring one leg back far enough, which causes a limp. Their stance phase is shortened and they do not let their foot go back much past the stance position shown in Figure 2(b). Without a correct toe off, they are unable to push off effectively, thus they cannot efficiently propel themselves forward. The GEM shoe can establish better walking patterns in two ways.

The GEM shoe can assist patients by equalizing the forward progression on each step. Wearing the GEM shoe on the strong foot will decrease the forward progression of the strong leg. In effect, both legs will propel the individual forward by a similar amount. This method is effectively compensating for the incorrect gait and will not rehabilitate the patient. Compensating for the incorrect gait would be useful for individuals who cannot benefit from rehabilitation.

Another method will rehabilitate the patient by exaggerating the limited forward motion of the weak leg and stimulating correct gait. Exaggerating the gait impairment is one of the methods used for rehabilitation on the split-belt treadmill $[14,15]$. Similar to the split-belt treadmill, the individual's small forward motion on the weak leg is further reduced, but in this case by wearing the GEM shoe on the weak-side foot. The reduced step size motivates the individual to beneficially lengthen their stride. The learned motion leads to after-effects close to the correct gait. Also, the backward motion of the shoe causes the foot to move backward, closer to the ideal location for toe off. Through repeated sessions, a gait impaired individual should achieve a lengthened stride and be able to place their foot in the proper location to effectively push off while walking over ground.

\subsection{Foot Haptics}

Several devices currently exist that can display forces and motions to to the feet. The HapticWalker [17] provides two foot platforms connected to a stationary machine that can simulate many walking environments, such as stairs and rough ground surfaces. Otis et al. [10] developed a locomotion interface allowing individuals to walk freely around a virtual environment while still remaining in the confines of the workspace.

Many studies have examined the design of prosthetics. The roll-over shape of prosthetics is particularly relevant to the GEMS designs. When using a prosthetic, the roll-over shape should match the motion of a non-disabled foot while walking [3]. Rietman et al. [16] provide of summary of many aspects of foot prosthetic devices.

\section{Shoe Design}

The GEM shoe consists of three parts: the rear wheel, the middle roller, and the front toe. These three parts work together to move the foot backward throughout the gait cycle. Figure 4 shows an idealized motion of the shoe. When initial heel contact occurs, the downward force causes the rear wheel to move the foot backward. During mid-stance, the middle roller allows the foot to move backward as the wearer begins to push their body forward. Finally, the front toe will contact the ground, allowing the wearer to push off as they normally would when wearing a shoe.

\subsection{Rear Wheel}

The rear of the shoe is designed to push the foot backward as the heel comes in contact with the ground. Figure 5 shows one of the two symmetric sides of the mechanism. In order to provide stability, there are two wheels, two gears, two racks, and two ball bearings connected to one axel. The gears mesh with the racks to achieve a backward force as shown in Figure 6. The bearings roll along the opposite side of the racks ensure the gears maintains contact with the racks. When the wearer steps down onto the rear of the shoe, the rear wheels contact the ground and the racks move down causing the gears to spin. Specifically, the downward force on the shoe is applied to each rack, which exerts a force on one side of each gear and causes the gear and attached wheels to rotate backward. After the rack has descended, the middle wheel will come into contact with the ground. At the end of the step, gravity will pull the rear wheel back to the bottom of the rack and reset its position for the next step. 


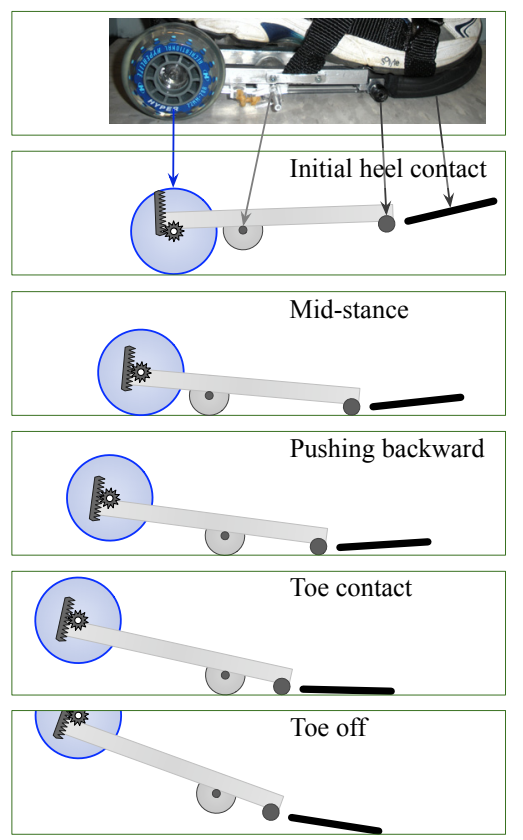

Figure 4: The GEM shoe causes backward motion of the foot throughout the stance phase. Initial heel-contact: The rear wheel translates the wearer's downward force into a backward motion. Midstance: The rear wheel will move up and the middle roller will contact the ground. Pushing backward: The middle roller will allow the wearer's backward force to cause a backward motion. Toe contact: As the middle roller rolls toward the front of the shoe, the shoe will tip forward. Toe off: The wearer will push off and swing their leg forward.

It is important to select the correct ratio between the gear size $\left(d_{g}\right)$ and the wheel size $\left(d_{w}\right)$ since there is a tradeoff between the horizontal force the rear wheel can exert $\left(F_{h}\right)$ and the horizontal distance the wheel can roll $\left(L_{h}\right)$ for a given downward force $\left(F_{v}\right)$ and height of travel $\left(L_{v}\right)$ as shown in (1) and (2).

$$
\begin{aligned}
& \frac{F_{v}}{F_{h}}=\frac{d_{w}}{d_{g}} \\
& \frac{L_{h}}{L_{v}}=\frac{d_{w}}{d_{g}}
\end{aligned}
$$

As the gear ratio $\left(d_{w} / d_{g}\right)$ increases, the total distance traveled increases linearly, but the total force decreases non-linearly as shown in Figure 7. Figure 7 assumes a person that exerts a downward force of $600 \mathrm{~N}$, which roughly matches the forces shown in Figure 3. The horizontal and vertical forces scale together, so a larger force downward, from a heavier person, would cause a proportionally larger backward force. Similarly, the forward force during the first half of the stance phase also increases with a heavier person.

In order for the shoe to cause a backward motion, the resulting force needs to be larger than the forward force applied during walking. For our prototype, the vertical distance, $L_{v}$, was $2.5 \mathrm{~cm}$ and we chose a ratio of $4.8: 1$, which results in a peak force of about $120 \mathrm{~N}$ and a maximum distance of $12 \mathrm{~cm}$. From our experiments, we found that this ratio was too small since the horizontal force caused the foot to travel backward too quickly and the foot stopped before transitioning to the middle wheel. A larger ratio would allow a smoother transition and provide a larger motion backward. However, too large of a ratio will provide too small of a backward force so the initial backward motion would occur later in the step.

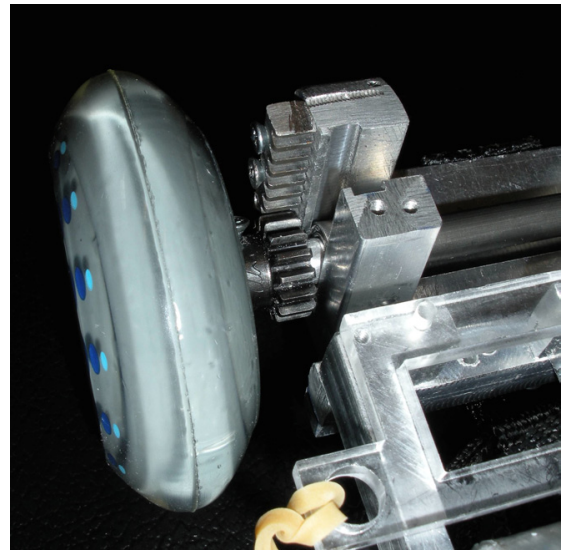

Figure 5: One of the two rear mechanisms that cause a backward motion when the wearer first places their foot on the ground.

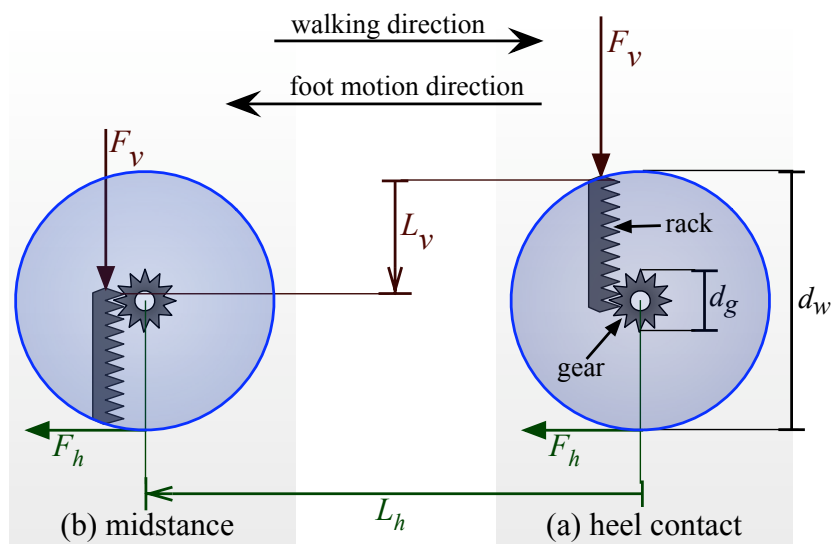

Figure 6: A downward force $\left(F_{v}\right)$ causes the wearer's foot to move backward by $L_{h}$. As the wearer pushes down on the heel of the shoe, the force is transmitted to the rack. The rack pushes on the gear, which causes the shoe to move backward (from right (a) to left (b)). The wearer is traveling to the right, but the foot is moving to the left while in contact with the ground.

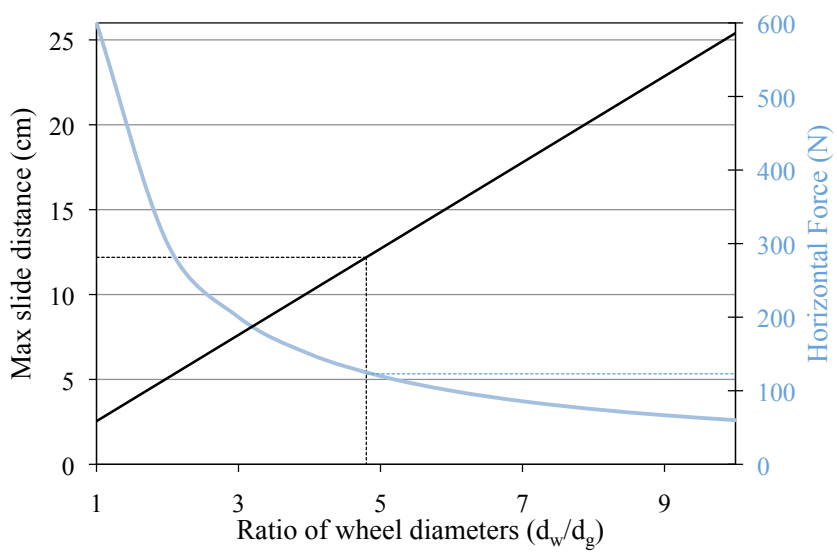

Figure 7: The ratio between the rear wheel and the gear affects the horizontal force and distance traveled. The forces scale linearly since the forces are based on the exerted force from the wearer, so a lighter person will feel the same ratio of downward to backward force as a heavier person. 


\subsection{Middle Roller}

The middle of the shoe, shown in Figure 8, allows the foot to move relative to ground over a definable distance using the backward force from the wearer. The concept is similar to a roller bearing connecting the shoe and the ground, but this roller has two diameters as shown in Figure 9. The large diameter contacts the ground and the smaller one contacts the frame of the shoe. Multiple diameters allow the roller to move different distances relative to the shoe and to the ground, which allows a longer low friction travel distance within the limited confines of the shoe.

The maximum motion of the shoe relative to ground is given by

$$
L_{g}=L_{s}\left(\frac{d_{l}}{d_{s}}+1\right)
$$

where $L_{g}$ is the maximum distance the shoe can move relative to the ground, $L_{s}$ is the distance the roller can move inside the shoe, and $d_{l}$ and $d_{s}$ are the large and small diameters of the roller, respectively, as defined in Figure 9. The maximum motion of the shoe should not exceed the stride length of the wearer. If the wearer's foot were to move backward more than their stride length, the resulting gait would be extremely awkward and this would likely invoke recovery reflexes. For the prototype, we chose a large diameter of $2.5 \mathrm{~cm}$, a small diameter of $0.5 \mathrm{~cm}$, and $L_{S}$ to be $10 \mathrm{~cm}$ so the entire foot has a maximum possible motion of $60 \mathrm{~cm}$, shown in Figure 10. In actual use, the travel distance is about half the theoretical maximum distance since the roller does not reach the front of the shoe.

Two springs are attached between the roller and the rear of the shoe. These springs serve two functions. First, they return the roller to the rear of the shoe at the end of each step. Second, they exert an increasing force throughout the back portion of the step. The increasing force is important since the wearer's horizontal force increases throughout the end of the stance phase. Without an increasing resistance force from the spring, the foot would rapidly accelerate backward. The spring provides a resistance so the wearer can prepare for toe off.

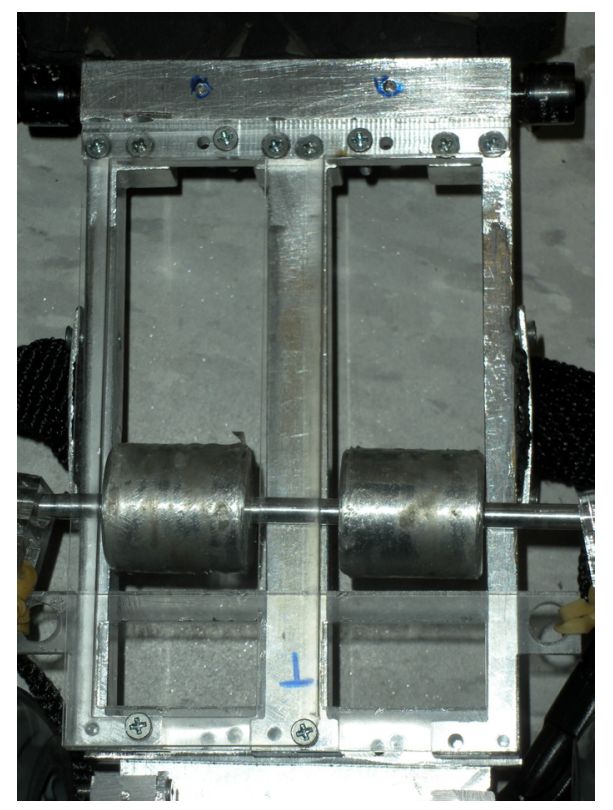

Figure 8: The bottom of the shoe showing the two diameters of the middle roller. The small diameter contacts the frame of the shoe and the large diameter contacts the ground. The two diameters of the roller allow the shoe to move a large distance relative to the ground while the roller only moves a small distance relative to the shoe while providing minimal friction.
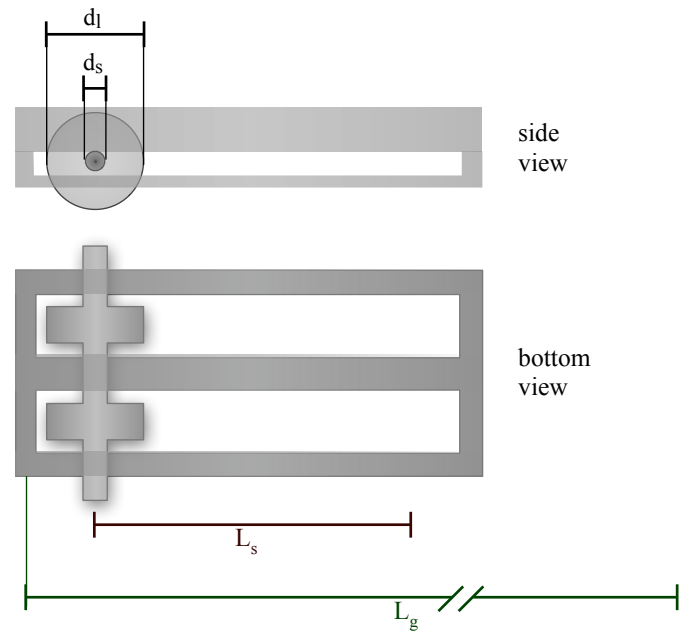

Figure 9: The middle roller allows the foot to move backward using the wearer's own forces. The roller has two diameters to allow the GEM shoe to move backward a large amount while the roller slowly moves toward the front of the shoe.

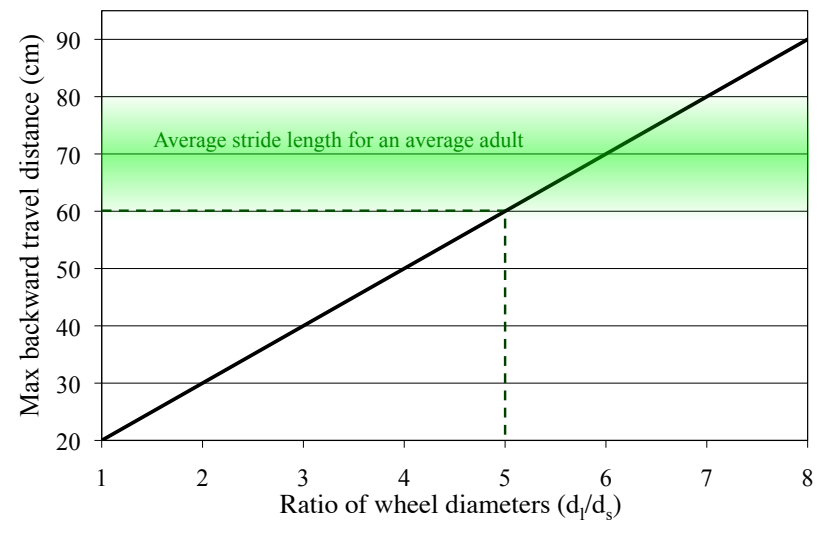

Figure 10: A larger ratio of the two middle roller diameters $\left(d_{l}\right.$ and $d_{s}$ ) increases the maximum travel distance. The roller motion inside the shoe is held constant in this design $\left(L_{s}=10 \mathrm{~cm}\right)$. The maximum travel distance should be less than the stride length of the wearer.

The roller moving forward relative to the shoe provides two beneficial properties. The roller moves with the wearer's center of pressure on the foot. As a person progresses through the stance phase, the center of pressure on their foot moves forward from their heel toward their toes $[8,22]$. The roller moving toward their toes ensures that solid contact is maintained between the shoe, roller, and ground. Second, as the roller moves forward, the shoe tilts forward slightly since the roller is taller than the front wheel. The shoe rotates around the front wheel and causes the solid portion at the front of the shoe to slowly come into contact with the ground, which allows for a smooth transition to the toe off stage of walking.

\subsection{Front Toe}

The front portion of the shoe consists of a free roller and a solid surface with friction. The free roller provides a low friction bearing that provides stability while allowing the rear and middle sections to cause backward motion. The front of the shoe is much like any other shoe, allowing the wearer to push off as they normally would during walking. The middle and rear portion of the shoe is rigid to support the forces required during walking, while the front of the shoe is flexible to allow the front of the wearer's foot to bend. 


\section{Results}

The three parts of the shoe (rear wheel, middle roller, and front toe) combine to cause the foot to move backward during contact with the ground. Figure 11 and the accompanying video show each of the three parts of the shoe in use.

In early experiments, we found the rear mechanism would provide about $8 \mathrm{~cm}$ of motion backward. The design concept works as intended, but the prototype could not handle extended testing since the gears and racks were spaced slightly too far apart. Due to the gap, the rear gears would skip causing decreased and bumpy motion. Since the prototype was unreliable, the remaining experiments were performed on the middle portion without the rear mechanism. We replaced the rear wheel and rack with a solid rubber section, so the rear section is much like the heel of an ordinary shoe. A second prototype of the same design has since been built and successfully walked on for over an hour without failing.

We used an Optotrak (Northern Digital, Waterloo, ON) motion tracking system to gather data on a subject walking over ground using only the middle portion of the shoe. For normal walking without gait modification, each leg would show the same results on the following metrics. Any deviation from symmetry indicates the effectiveness of the shoe in changing the gait pattern. We will refer to the foot with the GEM shoe on as the 'GEMS foot' and the other foot as the 'plain foot'.

One metric for examining gait is the length of each step. Wearing the GEM shoe decreased the step length, as shown in Figure 12. The step length of the plain foot is around 40 to $55 \mathrm{~cm}$, about $50 \%$ higher than the roughly $30 \mathrm{~cm}$ step length of the GEMS foot. Most of this difference arises from the GEMS foot moving backward during the stance phase. Essentially, the wearer is taking a half step backward for every two steps forward. The plain foot step length
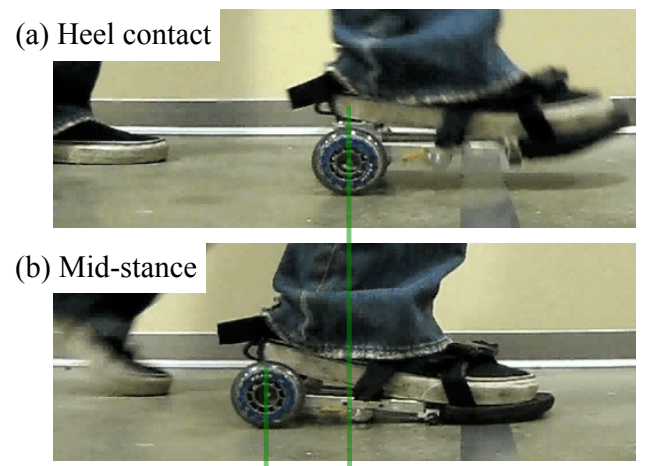

(c) Toe off

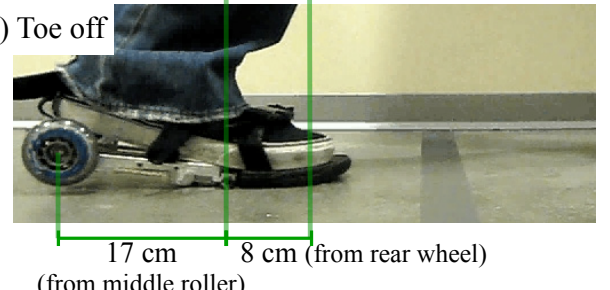

Figure 11: Our prototype of the GEM shoe caused a backward motion of around $25 \mathrm{~cm}$. (a) Upon initial heel contact, the rear wheel causes the foot to move backward by $8 \mathrm{~cm}$. (b) As the heel moves down, the middle roller comes into contact with the ground. The wearer then propels themselves forward and the roller allows the foot to move backward by $17 \mathrm{~cm}$. As the foot moves backward, the middle roller moves forward relative to the shoe and the angle of the shoe tips forward. (c) The front solid portion will touch the ground and prevent further backward motion and the wearer can push off into the swing phase. also decreased since the gait cycle is shortened so the wearer has less time to place the plain foot in the typical location.

The total backward distance traveled by each foot is shown in Figure 13. The GEMS foot moved more than $25 \mathrm{~cm}$ backward on most steps. Since the shoe is passive, we expected variations on each step and there is no guarantee that each step will provide the exact same motion. Slight differences in the exerted force and walking speed can change the distance traveled, which can be beneficial for gait rehabilitation [18]. For certain motions, external variations can increase limb impedance [13]. Higher limb impedance can help reduce the effect of perturbations, which will help the person's stability when walking without a corrective device. The plain foot also shows a slight backward motion, which is typical since the toe can move slightly as the heel contacts the ground and the push off force can cause the shoe to slip backward slightly. The GEMS results indicate a similar effect as the the splitbelt treadmill did when the treads were locked at a $2: 1$ ratio.

Another measure to classify walking is the percent of time in stance phase. Figure 14 shows a significant difference in the stance time between the foot with and without the GEM shoe. The wearer

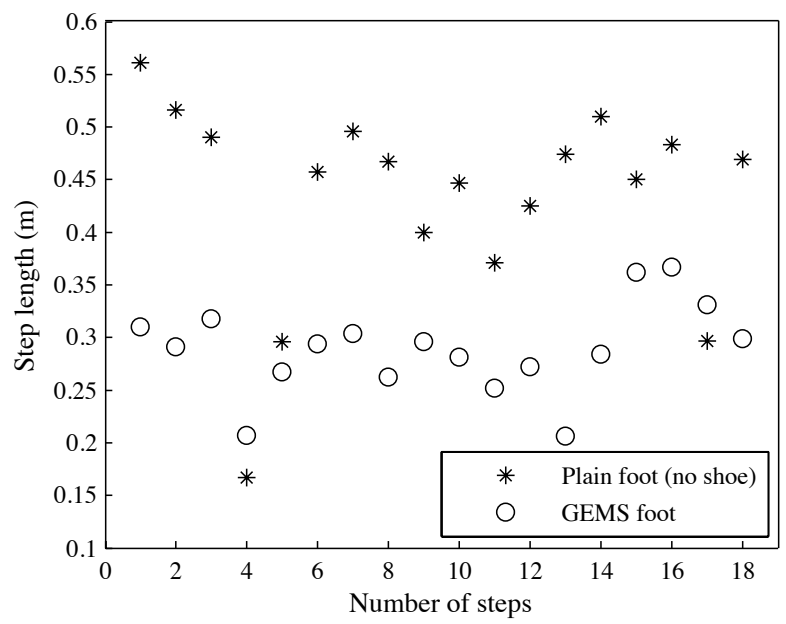

Figure 12: The GEM shoe shortened the total forward motion of the GEMS foot from the wearer's nominal $65 \mathrm{~cm}$ step length to roughly $30 \mathrm{~cm}$. The plain foot step length also decreased slightly.

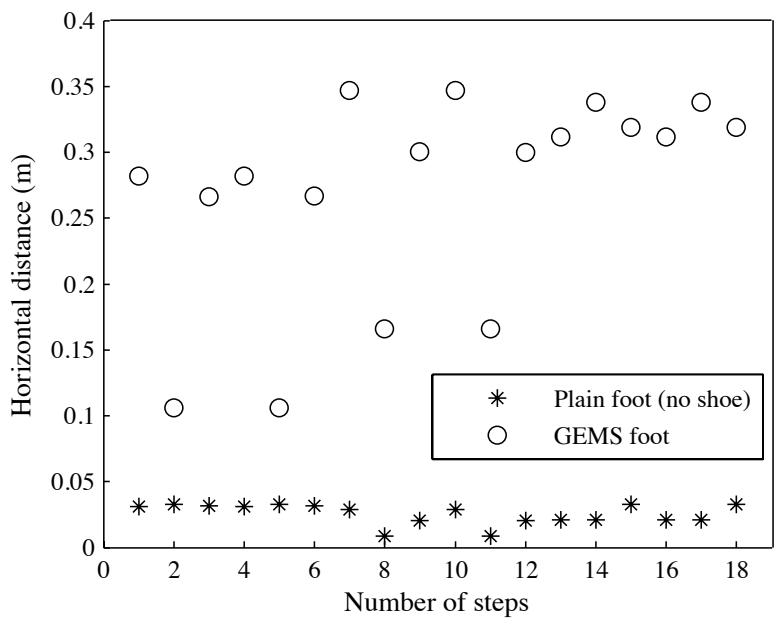

Figure 13: The GEM shoe enabled the wearer's foot to travel backward around $30 \mathrm{~cm}$ on many steps. With a typical shoe, the foot will move a few $\mathrm{cm}$ due to small movements as the heel and toe contact the ground. 


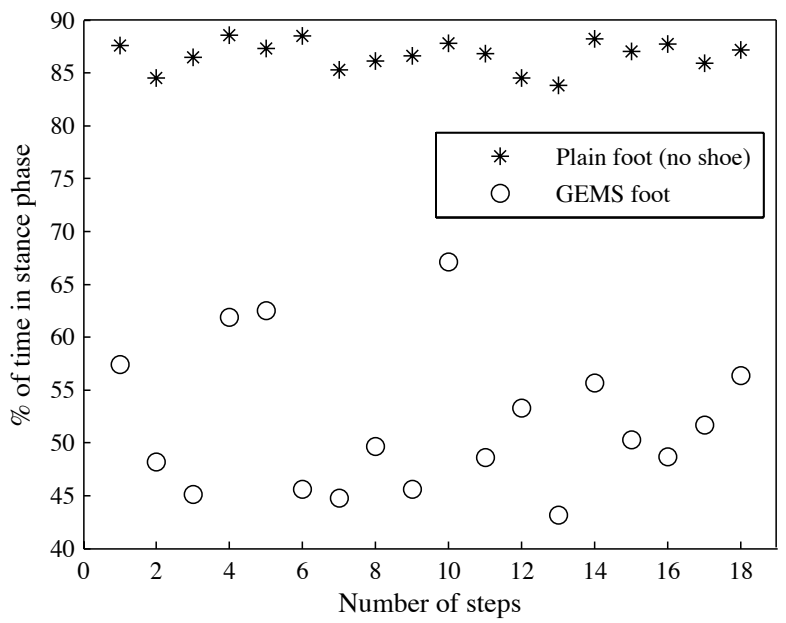

Figure 14: The GEM shoe reduced the time in stance phase since the GEMS foot traveled backward and the wearer began toe off earlier.

is placing the plain foot on the ground longer than the GEM foot. Since the GEMS foot is moving backward, the stance phase is effectively shortened.

These results indicate that the GEM shoe can dramatically alter the walking pattern of the wearer. The altered gait for our shoe looks similar to those from a person walking on a split-belt treadmill $[14,9,15]$. The modified gait is promising, but further experiments on long term effects are necessary.

\section{Conclusions}

We have demonstrated the potential of the GEM shoe to alter normal gait patterns. The modified gait patterns are similar to those found in subjects walking on a split-belt treadmill, but the shoe has several important benefits. The shoe allows the wearer to walk over ground, a natural walking environment, and the shoe is passive, deriving all necessary force from the wearer. The GEM shoe can also provide additional rehabilitation in more locations, even in the comfort of one's own home.

Further experiments are necessary to determine how well the modified gait will transfer to walking without the GEM shoe. With the rebuilt rear portion of the shoe, we can conduct extended studies using the combined effects of the middle and rear portions of the shoe. We can determine the parameters necessary to achieve a correct and smooth perturbation throughout the entire step. To further understand the effect this shoe has on gait, we can also analyze the wearer's center of mass and how rolling impacts the pendulum-like nature of walking.

There are many possible permutations to test transfer of the modified gait. One simple test would be simply to walk with the shoe for a period of time and then remove it and test the after effects. Another possible scenario would be to train an individual on the split-belt treadmill and then put the shoe on them when they walk over ground. The wearer will experience similar motions on the treadmill and the ground, but the context will change. Ideally, many of the motions will transfer to the new context.

\section{ACKNOWLEDGMENTS}

The authors thank Allison Okamura for an exchange of ideas. The authors also thank Amy Bastian, Erin Vasudevan, Paxson Trautman, and Noah Cowan for their help. This work was funded by NSF grant REU-0649069, NSF grant EEC-9731748, and Johns Hopkins University.

\section{REFERENCES}

[1] A. Gordon, G. Westling, K. Cole, and R. Johansson. Memory representations underlying motor commands used during manipulation of common and novel objects. J. Neurophysiol, 69(6):1789-1796, 1993.

[2] R. Grasso, L. Bianchi, and F. Lacquaniti. Motor Patterns for Human Gait: Backward Versus Forward Locomotion. J. Neurophysiol, 80(4):1868-1885, 1998

[3] A. Hansen, D. Childress, and E. Knox. Prosthetic foot roll-over shapes with implications for alignment of trans-tibial prostheses. Prosthetics and Orthotics International, 24(3):205-215, 2000.

[4] R. Johansson. Sensory input and control of grip. Sensory guidance of movement: Novartis Foundation Symposium, 218:45-59, 1998.

[5] R. Keamey. Context dependence of intrinsic and reflex contributions to ankle stiffness. In Proc. of 1st International IEEE EMBS Conf. on Neural Engineering, pages 434-437, March 2003.

[6] J. Krakauer, M. Ghilardi, and G. Ghez. Independent learning of internal models for kinematic and dynamic control of reaching. Nature Neuroscience, 2:1026-1031, 1999.

[7] T. Lam, M. Anderschitz, and V. Dietz. Contribution of feedback and feedforward strategies to locomotor adaptations. J. Neurophysiol, 95:766-773, 2006.

[8] R. Mann, J. Hagy, V. White, and D. Liddell. The initiation of gait. J. Bone and Joint Surgery, 61:232-239, 1979.

[9] S. Morton and A. Bastian. Cerebellar Contributions to Locomotor Adaptations during Splitbelt Treadmill Walking. J. Neurosci., 26(36):9107-9116, 2006.

[10] M. Otis, C. du Tremblay, F. De Rainville, M. Mokhtari, D. Laurendeau, and C. Gosselin. Hybrid control with multi-contact interactions for $6 \mathrm{DOF}$ haptic foot platform on a cable-driven locomotion interface. In Symposium on Haptic Interfaces for Virtual Environments and Teleoperator Systems, pages 161-168, March 2008.

[11] P. Perkins and M. Wilson. Slip resistance testing of shoes - new developments. Ergonomics, 26(1):73-82, 1983.

[12] M. Redfern, R. Cham, K. Gielo-Perczak, R. Grönqvist, M. Hirvonen, H. Lanshammar, M. Marpet, C. Pai, and C. Powers. Biomechanics of slips. Ergonomics, 44(13):1138-1166, 2001.

[13] D. Reinkensmeyer, J. Emken, and S. Cramer. Robotics, motor learning, and neurologic recovery. Annu. Rev. Biomed. Eng., 6:497525,2004

[14] D. Reisman, R. Wityk, and A. Bastian. Split-belt treadmill walking adaptation in post-stroke hemiparesis. J. Neurologic Physical Therapy, 29:196, 2005.

[15] D. Reisman, R. Wityk, K. Silver, and A. Bastian. Locomotor adaptation on a split-belt treadmill can improve walking symmetry post-stroke. Brain, 130(7):1861-1872, 2007.

[16] J. Rietman, K. Postema, and J. Geertzen. Gait analysis in prosthetics: Opinions, ideas and conclusions. Prosthetics and Orthotics International, 61(1):50-57, 2002

[17] H. Schmidt, S. Hesse, R. Bernhardt, and J. Krüger. HapticWalker - a novel haptic foot device. ACM Trans. on Applied Perception, 2(2):166-180, 2005

[18] H. Shimada, S. Obuchi, T. Furuna, and T. Suzuki. New intervention program for preventing falls among frail elderly people. American Journal of Physical Medicine \& Rehabilitation, 83(7):493-499, 2004.

[19] L. Tesio and V. Rota. Gait analysis on split-belt force treadmills validation of an instrument. Amer J. Phys Med Rehabil, 87:515-526, 2008.

[20] H. van Hedel, B. Wirth, and V. Dietz. Limits of locomotor ability in subjects with a spinal cord injury. Spinal Cord, 43:593-603, 2005.

[21] M. Whittle. Gait Analysis: An Introduction. Elsevier Health Sciences, 1998.

[22] D. Winter. Human balance and posture control during standing and walking. Gait and Posture, 3:193-214, 1995.

[23] D. Winter, A. Patla, F. Prince, M. Ishac, and K. Gielo-Perczak. Stiffness control of balance in quiet standing. J. Neurophysiol, 80:1211-1221, 1998.

[24] J. Yang and M. Gorassini. Spinal and brain control of human walking: Implications for retraining of walking. Neuroscientist, 12:379, 2006. 